\title{
The Impact of a Pediatric Acute Unit in Reducing Length of Stay: A Success Story
}

\author{
Angela Caswell ${ }^{1}$, Hamad Al Khalaf ${ }^{2}$, Abdullah Al Mutrafy ${ }^{3}$, Rahayu Abd Rashid ${ }^{4}$ \\ ${ }^{1}$ Department of Nursing King Abdullah Specialized Children's Hospital, Riyadh, University of South Wales, South Bank University, London, \\ United Kingdom \\ ${ }^{2}$ Department of Pediatrics and Bed Management King Abdullah Specialized Children's Hospital, Riyadh University of Ottawa, Ottawa, \\ Canada \\ ${ }^{3}$ Department of Medical Services, King Abdullah Specialized Children's Hospital, Riyadh King Saud Bin Abdulaziz University for Health \\ Sciences, Riyadh, Saudi Arabia \\ ${ }^{4}$ Department of Pediatric Emergency, King Abdullah Specialized Children's Hospital, Riyadh University of Huddersfield, Huddersfield, \\ United Kingdom
}

\section{Email address:}

atcaswell@gmx.com (A. Caswell),h.a.alkhalaf@gmail.com(H. Al Khalaf),msded-kasch@NGHA.MED.SA (A. Al Mutrafy), ayumandrick@hotmail.com (R. A. Rashid)

\section{To cite this article:}

Angela Caswell, Hamad Al Khalaf, Abdullah Al Mutrafy, Rahayu Abd Rashid. The Impact of a Pediatric Acute Unit in Reducing Length of Stay: A Success Story. American Journal of Nursing Science. Vol. 8, No. 6, 2019, pp. 288-293. doi: 10.11648/j.ajns.20190806.11

Received: August 9, 2019; Accepted: August 24, 2019; Published: October 10, 2019

\begin{abstract}
Challenges to patient flow within acute hospital settings has been acknowledged internationally as a growing concern that has the potential to impact upon patient safety, satisfaction and organizational budgetary constraints. The development and introduction of a unique Pediatric Acute Unit pilot program has shown promising results which support its continued implementation. Dynamic in its approach, it has achieved a streamlined flow process, reduced overall length of stay, saved bed days (557.81) and reduced cost $(1,158,377$ SAR over 4 month pilot phase capturing the seasonal surge period of winter months). Using a mixed method of Plan Do Study Act (PDSA), including quantitative data collection, the team mapped the pilot study through 10 defined cycles of testing, evaluation and refinement. The (PAU) must be examined as a concept set against an overarching patient flow methodology which is part of a whole systems approach underpinning quality of care during the patient's journey.
\end{abstract}

Keywords: Acute Unit, Short Stay, Admission Rate, Pediatric Acute Unit

\section{Introduction}

King Abdullah Children's Specialized Hospital was commissioned during the summer of 2015. A quaternary university teaching hospital located in Saudi Arabia, comprising 600 beds within its vast campus.

Since then, attendance to the Emergency Department has witnessed an annual increase of (ED) attendance of 28\%; and inpatient admissions of 8,000. Demands upon existing services presented significant challenges related to the availability of inpatient beds. The organization shared an international -shared challenge which tested the ability to successfully manage demands placed upon the capacity of inpatient beds, manpower deficits, and the resulting impact of overcrowding this had upon the Emergency Department.

Delays for patients receiving timely allocation of inpatient beds, increased length of stay and associated escalation of costs formed the fundamental platform upon which this major quality improvement project was developed.

A trend analysis was undertaken during the 6 months of the previous winter seasonal surge period. The average length of stay (LOS) between 4-8 days. Patients who were discharged following short stay periods ranging between 24-48 hours were examined and totaled 1092 over the selected period (Table 1). These cases were identified as a potentially selected group who might be considered for management in the (PAU).

Key stakeholders prepared a proposal to utilize an existing area located adjacent to the Pediatric Emergency Department (ED) for the Pediatric Acute Unit (PAU). This was comprised 
of a 10 bed unit and was staffed by nurses from the (ED). The planned time for the pilot to be conducted was December March which aligned with an identified winter surge period. The proposal was therefore cost neutral but had the potential to result in a significant cost reduction through a streamlined patient flow process; saving bed days and manpower resources.

Limited research is currently available in relation to similar pediatric units whilst a plethora of literature is available for the adult population $[1,2]$. This presented a challenge related to available internationally benchmarked statistics against which performance outcomes of the (PAU) could be measured.

Key objectives of the (PAU) were to optimize safety through efficiency and streamlined processes. Agreed guidelines and the development of a final Departmental Policy and Procedural document (DPP) was developed that would guide and support healthcare staff in the admission and exclusion criteria of patients who would be eligible for management in the unit [3-5]. The range of accepted conditions had been acknowledged by Macy et al, 2010 [6] as representing a significant number of hospital admissions among pediatrics.

Effective observation, assessment, re-assessment and rounding between nurses and physicians in the units were essential to maintain optimal safety and expedite robust management and early discharge home [7]. The psychology underpinning the concept of the (PAU) was for a brief stay without the expectation of admission to the ward. Parents would receive explanation related to the methodology of the unit which was designed to support safe and timely discharge.

Return visits requiring admission was examined by Bardach et al, 2013 and noted an overall 3\% rate. They conducted a retrospective study, with sample size $(n=44,097)$ pediatric patients who visited a tertiary teaching hospital. Trends of revisits and admissions were noted to be from aged group 0-7 years; $44.1 \%$ of which represented with same original symptoms and $76 \%$ related to respiratory conditions. This study was selected as a comparator to the geographical location, climate and seasonal surge of respiratory related conditions in the young. With the pilot study hospital's average of 8,000 inpatient admissions annually, of which $25 \%$ were related to respiratory conditions, predominantly bronchiolitis, this study was used as a comparator.

During an earlier study, Gouin et al, 1997 [8], identified that, following the introduction of similar pediatric observational, short stay units, the rate of admissions for a similar group had decreased by $23 \%$. A retrospective review of pediatric respiratory patients who had previously been identified as requiring inpatient admissions had been reduced from $9.5 \%$ to $4.2 \%$ along with an overall reduction in length of stay $[9,10]$.

Physician satisfaction with the introduction of similar units was the subject of a quantitative study by Rentz et al, 2004 [11]. A sample size of $(n=248)$ was selected using a randomized approach; the response to the questionnaires distributed was $80 \%$. Using the Likert scale $1-4$, more than $60 \%$ stated that they were satisfied with the implementation of a pediatric observation unit for common conditions such as asthma, gastroenteritis etc.

\section{Method}

The team comprised of key stakeholders examined ways in which performance could be measured. They outlined the following core areas as indicators:

a) Number of admissions from (PAU) following 72 hours

b) Number of return visits to the (ED) requiring hospitalization having been discharged from (PAU)

c) Direct feedback from patients and parents as end users of the (PAU)

d) Physician satisfaction

Clearly defined admission and exclusion criteria was developed and presented as a guideline for physicians to determine a patient's suitability for admission to (PAU).

Using Plan Do Study Act (PDSA) methodology (Prybutok, 2018), the team identified key areas for improvement and the associated challenges before the introduction of the pilot study.

The method took the team through a total of ten (10) PDSA cycles over a period of nine (9) months from August 2017 May 2018. Using the Pareto principle of cause and effect $20 / 80$ rule, the team ranked the main identified challenges through a system of Multivoting, as areas for immediate improvement through the (PAU) pilot project (Figure 2).

Clear guidelines were developed and refined prior to presentation to the executive committee to gain approval for the launch of the project.

\section{Guidelines}

Managed by Pediatric Department Physicians. 12 hour shifts covered by experienced consultants. Will undertake at least 3 rounds per shift (at least 4 hour intervals). The model will facilitate the timely discharge of patients $24 / 7$. Patients will be discharged day/night time and with the supervision/consultation of the most responsible physician.

Safety: International literature supports the safety and efficiency of the (PAU) model of care, facilitating earlier discharge of patients. This model will improve patient flow, reducing demands upon inpatient beds and (ED) capacity [12]. The PAU model has been proven to reduce non-socomial infections related to prolonged hospitalization. Similar units have demonstrated enhanced quality satisfaction and achieved better psychological outcomes for patients and parents [13, 14].

Impact of (PAU) on Hospital Resources: Optimal use of (PAU) has a positive correlation with efficient utilization of inpatient beds. The care model of (PAU) requires fewer handovers, is more protocol-driven and results in more frequent patient assessment, the rapid access to diagnostic facilities compared to the more traditional model of inpatient care. The application of lean methodology streamlines the process of diagnostics and management and results in reduced length of stay and early discharge. As the (PAU) is designed to allow overnight discharge, as opposed to the traditional model of inpatient care [15].

Impact of (PAU) on Trainee Education: International survey results during a rotational period on acute pediatric units indicated that the educational experience was favourable. 
Clustering of patients with symptoms suggestive of straightforward diagnoses enhanced the trainee's educational experience. Similarly, managing patients in (PAU) provides experience of the management of lower acuity patients.

Admission Criteria: Two main elements must be considered when selecting patients for transfer and management in (PAU).

a) Selecting cases with a definitive diagnosis with an estimated length of stay $<48$ hours.

b) Selecting cases with a low acuity.

Controlling these elements will lead to the appropriate decision to transfer to (PAU) with an expected rapid turnover rate. By moving away from a decompression capacity model, optimal use can be made of existing inpatient beds to reduce length of stay for patients in (ED).

Targeted cases that will be selected for transfer and management in PAU:

(1) Bronchiolitis in infants older than $>6$ months and requiring $<1 \mathrm{~L} / \mathrm{min}$ oxygen.

(2) Pneumonia in children $>3$ months and requiring < $1 \mathrm{~L} / \mathrm{min}$ of oxygen via nasal cannula.

(3) Bronchial asthma/viral induced wheezing or hyperactive airway disease in older than 6 months requiring oxygen $<1 \mathrm{~L} / \mathrm{min}$ via nasal cannula.

(4) Urinary tract infections in patients $>3$ months with no previous history of multidrug resistant organisms (MDRO's).

(5) Functional constipation without need for major work ups.

(6) Well looking infant (any age) with fever to rule out sepsis who are admitted for follow up of cultures.

(7) Gastroenteritis with isonatremic dehydration at any age who have exceeded the 12-16 hours observation within the (CDU) Clinical Decision Unit.

(8) Cases that would require admission for short period ( $<48$ hours) for intra-venous hydration or antibiotic administration e.g. pharyngitis with poor oral intake.

(9) Any condition in which the child is admitted primarily for short term (48hours or less) intra-venous (IV) hydration or IV antibiotics. e.g. pharyngitis with poor oral intake.

(10)Non-oncological cases that are admitted primarily for transfusion or short term IV therapy.

(11)Resolved anaphylaxis at any age that is kept for observation.

(12)Any stable medical case requiring observation not exceeding 48 hours. These must not have an acute medical care plan e.g. antidote infusion.

(13)Drug overdose/ingestion for which the child is admitted primarily for observation for 24-48 hours or less, and does not require an antidote infusion, e.g. (but not limited to):

Drug ingestions providing that:

a) Ingested amount is small.

b) Short acting type that does not require an infusion management apart from IV fluids.

c) That does not require cardiac monitoring.

Preliminary Results of Flow and Utilization
End of first month (December) review of data and analysis of challenges identified with corrective actions taken. 115 discharged from PAU, average length of stay (LOS) 22.7 hours. Overall bed days saved 133.4 equating to an estimated 333,680 SAR saving.

End of second month (January) review of data and analysis of challenges identified with corrective actions taken. 144 discharged from PAU, average LOS 27.9 hours. Overall bed days saved 198.4 equating to an estimated 496,187 SAR saving.

End of third month (February) review of data and analysis of challenges identified with corrective actions taken. 117 discharged from PAU, average LOS 25.1 hours. Overall bed days saved 131.4, equating to an estimated 328, 51 SAR saving.

End of fourth and final month (March). Analysis of overall pilot. Presentations of results and recommendations. 73 discharged from PAU, average LOS 27.7 hours. Overall bed days saved 94.4, equating to an estimated 235,000 SAR saving.

Using a modified questionnaire from that provided to physicians, nursing personnel were also involved with a sample size of twelve (12) selected using a random approach through email.

Parents and patients (depending on age applicability) were also invited to complete a satisfaction feedback questionnaire by placing copies on nearby areas with a sealed return box, assuring with anonymity for those choosing to take part. Of the 558 patients admitted to (PAU) during the pilot program, zero return rate was achieved. Conditions and related attendances to the (ED) of which were admitted to (PAU) were predominantly related to respiratory conditions. The highest rate $24.01 \%$ with bronchiolitis, secondly $17.95 \%$ with gastroenteritis (Table 3).

\section{Results}

\subsection{Epidemiology}

Age:

1. $0-1: 234$

2. 1-3: 157

3. 3-6: 86

4. Above 6: 79

Gender:

1. Male: 285

2. Female: 257

Since the start of the pilot study during December 2017 and its completion, March 2018548 patients discharged home with no return within 72 hours. 449 admitted to the ward as exceeded the cut off period of 72 hours, requiring longer management and observation. The average length of stay 25.9 hours. The overall beds days saved were 557.81 equating to an estimated cost saving of 1,158,370.00 Saudi Arabian Riyals (SAR) (Table 4).

The overall performance of the (PAU) was successful. A trend analysis of activity witnessed a slower start at the beginning of the pilot study related to lack of understanding, initial reservations among some staff and overall awareness of 
the program. The upward trend during January and February was linked to the seasonal surge in attendances as well as increased awareness and acceptance among key stakeholders. The overall results were extremely promising:

a) Bed days saved during pilot- 557.8

b) Discharges- 449

c) Cost saving- 1,158,377 SAR

The team wanted to achieve a 360 degree review of the (PAU) project from key stakeholders and end users. A series of questionnaires were distributed to physicians, nurses, patients and parents.

\subsection{Analysis of Questionnaires}

Using a Likert satisfaction score, questionnaires were distributed to all parents and patients for anonymous (de-identified) completion and submission using a mail box located in the unit. A response rates of completed questionnaires reached $75 \%$. Physicians (sample size $n=12$ ) were sent the Likert questionnaire via email.

Table 1. Results of Questionnaire from Physicians.

\begin{tabular}{ll}
\hline & Physician \\
\hline Efficiency & $75 \%$ \\
Safety & $25 \%$ \\
Cost Effectiveness & $41.60 \%$ \\
Education & $33.30 \%$ \\
Workload & $96.60 \%$ \\
\hline
\end{tabular}

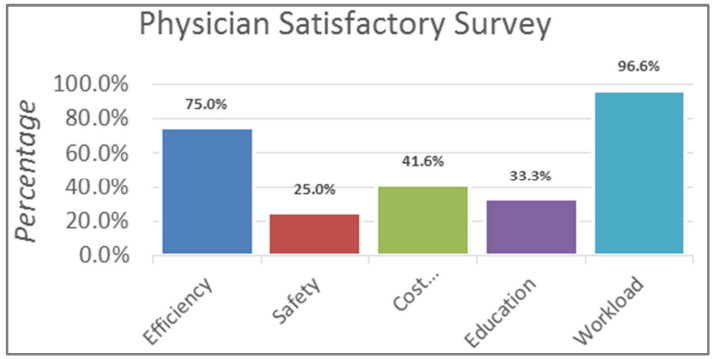

Figure 1. Physician Satisfactory Survey.

Table 2. Results of Questionnaire from Nurses.

\begin{tabular}{ll}
\hline & Nurses \\
\hline Efficiency & $85 \%$ \\
Safety & $90 \%$ \\
Cost Effectiveness & $87 \%$ \\
Education & $84 \%$ \\
Workload & $82 \%$ \\
\hline
\end{tabular}

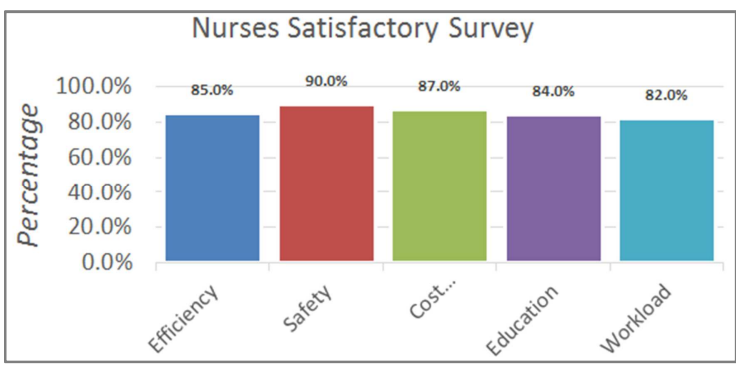

Figure 2. Nurses Satisfactory Survey.

Nursing satisfaction rating- using a Likert scale the sample size was balanced to correspond with that of the physician group and therefore $n=12$.

Using the domains:

1. Efficiency- $85 \%$ strongly agreed that the PAU model increased efficiency of patient flow and reduced delays.

2. Safety- $90 \%$ strongly agreed that the model ensured and maintained safety for patient and healthcare personnel.

Cost Effectiveness- $87 \%$ strongly agreed that the model was cost effective in reducing the demands on inpatient beds.

3. Education- $84 \%$ strongly agreed that this was an ideal opportunity to increase educational awareness by identifying patients most suitable for management in the PAU and added that this increased nurse empowerment along with strengthen dialogue with all key stakeholders.

4. Workload- $82 \%$ strongly agreed that the PAU model would reduce the workload on inpatient staff.

Table 3. Combined Questionnaire Results.

\begin{tabular}{lll}
\hline & Physician & Nurses \\
\hline Efficiency & $75 \%$ & $85 \%$ \\
Safety & $25 \%$ & $90 \%$ \\
Cost Effectiveness & $41.60 \%$ & $87 \%$ \\
Education & $33.30 \%$ & $84 \%$ \\
Workload & $96.60 \%$ & $82 \%$ \\
\hline
\end{tabular}

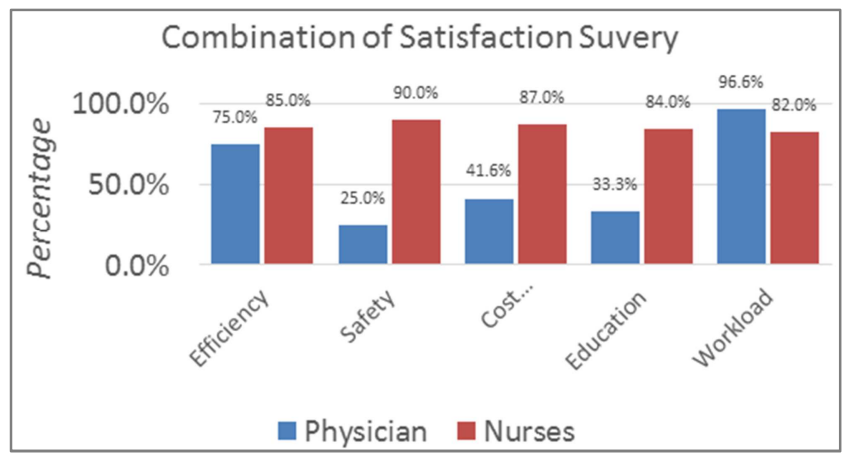

Figure 3. Combination of Satisfaction Survey.

Patient and parent satisfaction rating- the samples size of voluntary participants was 360 reflecting an overall satisfaction score as indicated in the chart (Figure 4). 75\% responded that they believed the overall experience of the PAU was excellent, $18 \%$ felt it was very good and $7 \%$ good. There were no negative comments or scores.

\section{Discussion and Conclusion}

Following a literature search limited research was available related to pediatric acute units. The concept appears novel for this patient population compared to adults. Initial slow engagement of key stakeholders reflected the need to prepare a more comprehensive educational /awareness campaign prior to its implementation. The pilot was noted to have achieved a $75 \%$ rating as excellent, and received no negative comments or ratings (Figure 6). The pilot project supported winter resilience planning measures and resulted in efficient, safe and timely patient management, reduced length 
of stay, patient satisfaction and a significant cost saving of $1,158,377.00$ (SAR) during the period of four (4) months (Table 5). With a reduced length of stay, the project saved 557.8 inpatient bed days and discharged 449 patients safely with no re-admissions. The (PAU) will be utilized as part of winter pressure preparation and has the potential to become an established full time, long term program. There is scope for further study focusing on acute respiratory illness in the pediatric population which this study demonstrated represented $46.39 \%$ which was supported by the earlier research conducted by Bardach et al, 2013 [16]. Related to the annual inpatient admissions. Future direction and conclusion to the project suggests there is scope to examine the feasibility of creating a specialized acute respiratory unit (Figure 5). This proposal could achieve a rapid access for this particular patient group and has the potential to achieve an overall reduction in length of stay. Health education and prevention should also be a feature of such a bespoke unit with an emphasis on prevention, for example, asthmatic patients.

Table 4. Discharge from the unit between 24-72 hours target time.

\begin{tabular}{llll}
\hline Discharge within 24/48 hours & 24 hours & $\mathbf{4 8}$ hours & $\mathbf{7 2}$ hours \\
\hline Oct-16 & 31 & 157 & 146 \\
Nov-16 & 32 & 162 & 171 \\
Dec-16 & 21 & 142 & 170 \\
Jan-17 & 22 & 146 & 147 \\
Feb-17 & 37 & 139 & 124 \\
Mar-17 & 37 & 166 & 195 \\
\hline
\end{tabular}

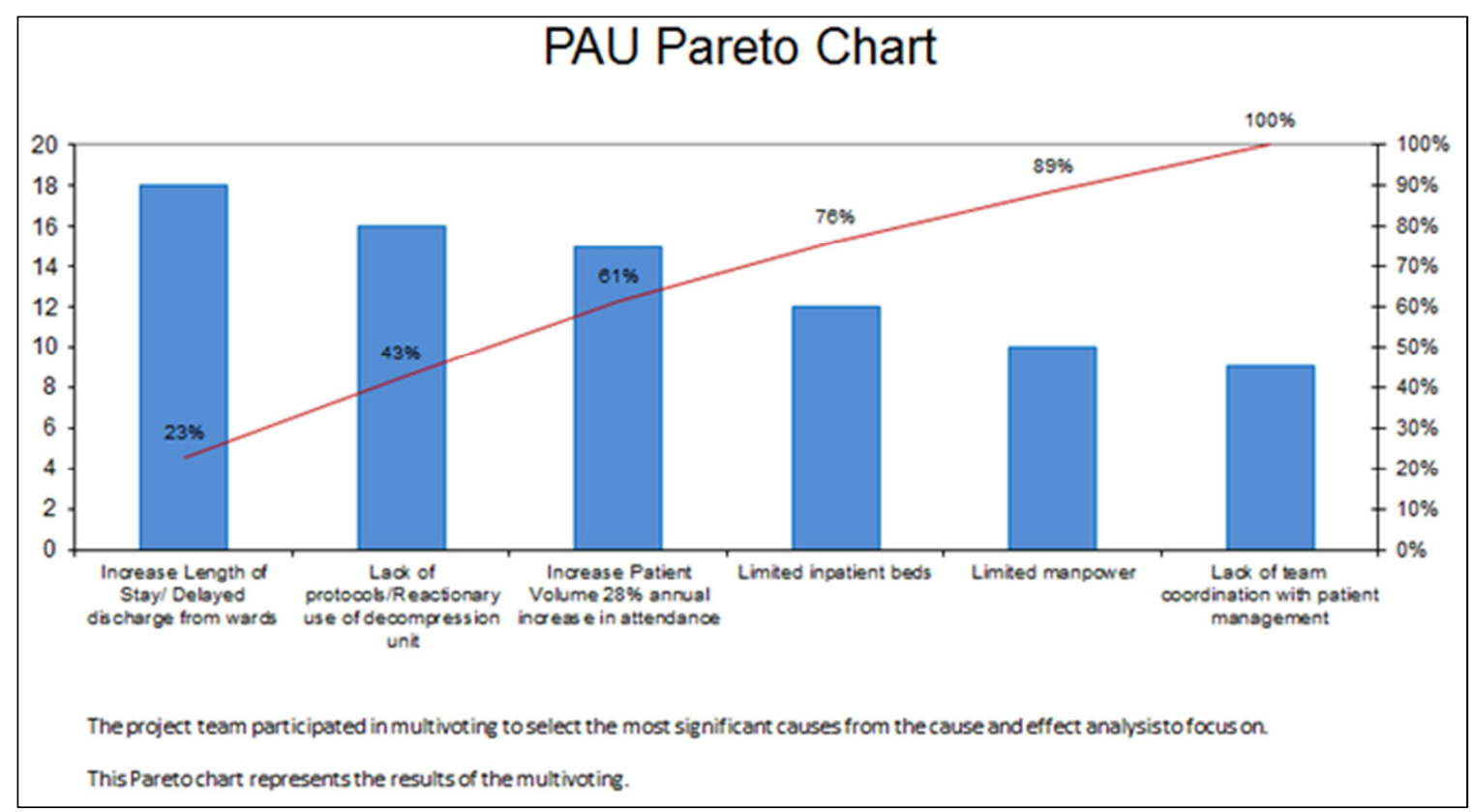

Figure 4. Pareto Chart.

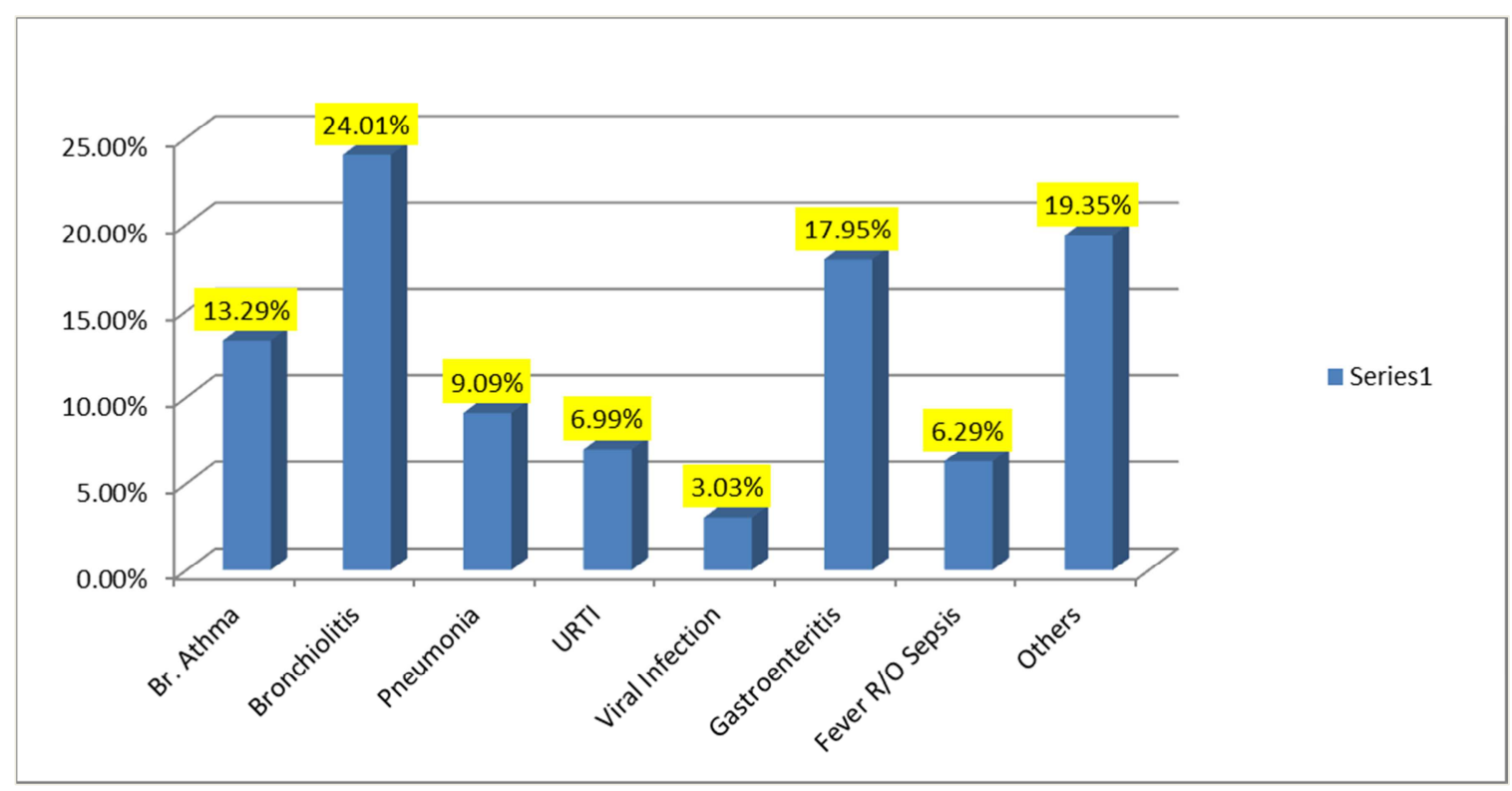

Figure 5. Admission Categories. 
Table 5. Overall tabulated performance results

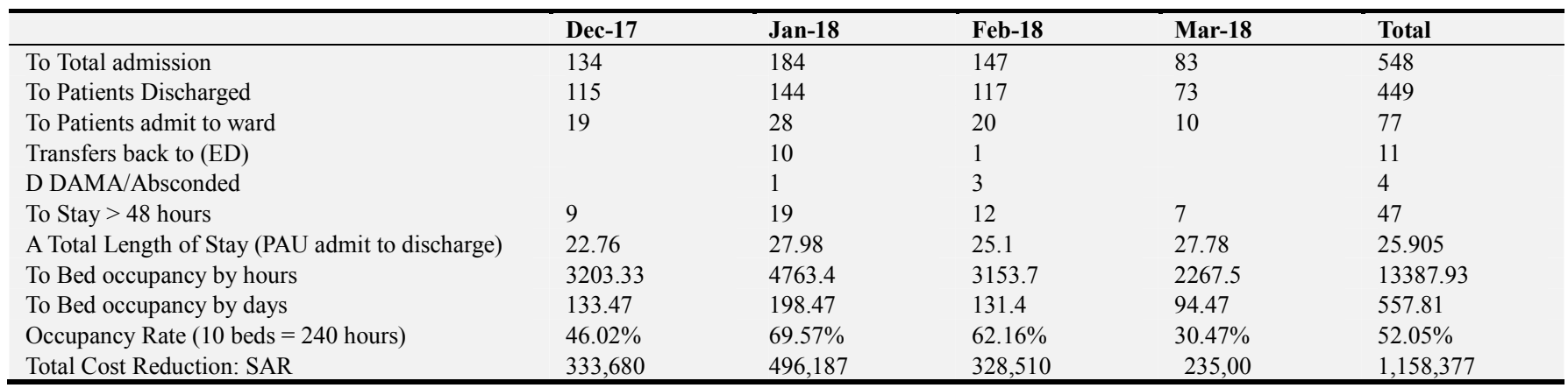

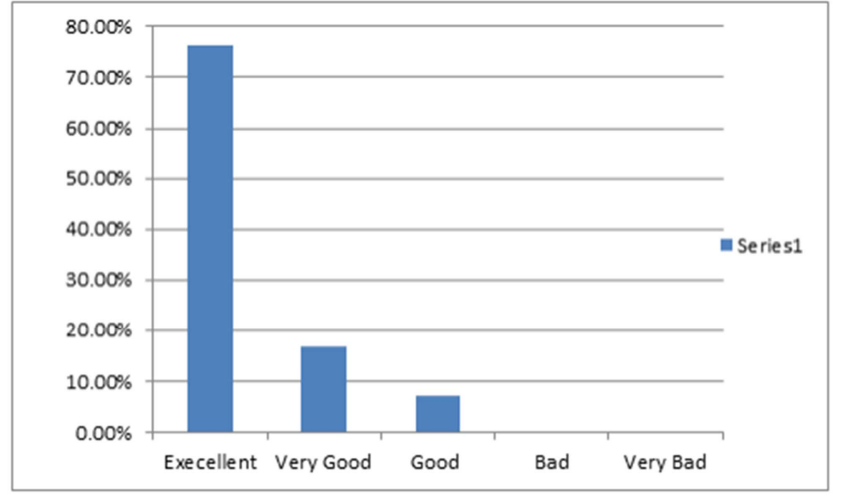

Figure 6. Patient Experience Survey.

\section{References}

[1] Graff, L. G. Dallara, J. Ross, M. A. Impact on the care of the emergency department chest pain patient from the chest pain evaluation registry (CHEPER) study. American Journal of Cardiology 199780 (5) p 563-568 1997).

[2] Ross, M. A. Graff, L. G. Presenting the observation unit and concept to administration and payors. Observation Units. Dallas Texas. American College of Emergency Physicians. 1998 p 6-15.

[3] Marks, M. K. Lovejoy, F. H. Rutherford, P. A. Impact of a short stay unit on asthma patients admitted to a tertiary pediatric hospital. Quality Management in Healthcare. 1997; 6 (1): p 14-22.

[4] Crocetti, M. T. Barone, M. A. Amin, D. D. Pediatric observation status beds on an inpatient unit: an integrated care model. Pediatric Emergency Care. 2004: 20 (1) p 17-21.

[5] Mallory, M. D. Kadish, H. Zebrack, M. Nelson, D. Use of a pediatric observation unit for children with dehydration caused by gastroenteritis. Pediatric Emergency Care.

[6] Macy, M. L. Kim, C. S. Sasson, C. Lozon, M. M. Davis, M. M.
Pediatric observation units in the United States: a systematic review. Journal of Hospital Medicine 2010; 5 (3) p 172-182 2010.

[7] Zebrack, M. Kadish, H. Nelson, D. The pediatric hybrid observation unit: an analysis of 6477 consecutive patient encounters. Pediatrics 2005; 115 (5).

[8] Gouin, S. Macarthur, C. Parkin, P. C. Schuh, S. Effect of a pediatric observation unit on the rate of hospitalization for asthma.

[9] Greenberg, R. A. Dudley, N. C. Rittichier, K. K. A reduction in hospitalization, length of stay and hospital charges for croup with the institution of a pediatric observation unit. American Journal of Emergency Medicine 2006; 24 (7): p 818-821.

[10] Miescieri, M. Nelson, D. Firth, H. Kadish, H. Children with asthma Admitted to a Pediatric Observation Unit. Pediatric Emergency care October 2005. Volume 21 Issue 10 p645-649.

[11] Rentz, A. Kadish, H. Nelson, D. Physician satisfaction With a Pediatric Observation Unit Administered by Pediatric Emergency Medicine Physicians. Pediatric Emergency Medicine July 2004. Volume 20. Issue 7. P 430-432.

[12] Noval, J. Campoamor, M. T. Avantas, E. Short stay medical units: an appropriate place to manage community acquired pneumonia? Annals of Medicine International 2006; 23: p 416-19.

[13] Broquetas, J. M. Pendrent, R. Martinez-Lorens, J. M. Short stay respiratory unit: a new option for inpatient care. Archivos de Bronconemolgia (3) 2008 p 252-56.

[14] Guirao-Martinez, R. Sempere- Selva, M. T. Lopez, A. Short stay medical unit, an alternative to conventional hospitalization. Revista Clinica Espanola 2008; 208 (5) p 216-21.

[15] Conners, G. P. Melzer, S. M. Committee on Pediatric Emergency Medicine 2012. Pediatric Observation Units. Pediatrics 130 (1): p 172-179.

[16] Bardach, N. S. Vittinghoff, E. Penalaza, R. A. Edwards, J. D. Yazdany, J. Lee, H. C. Boscardin, W. J. Cabana, M. D. Dudley, R. A. Measuring Hospital Quality Using Pediatric Readmission and Revisit R36ates. Pediatrics 2013132 (3) p 429-4. 